\title{
Free Energies of Proton-Coupled Electron Transfer Reagents and Their Applications
}

\author{
Rishi G. Agarwal*,1, Scott C. Coste, ${ }^{\dagger}, 1$ Benjamin D. Groff, ${ }^{\dagger, 1}$ \\ Abigail M. Heuer, ${ }^{\dagger, 1}$ Hyunho Noh, ${ }^{\dagger, 1}$ Giovanny A. Parada, ${ }^{\dagger, 1,2}$, Catherine F. Wise, ${ }^{1}$ \\ Eva M. Nichols, ${ }^{3}$ Jeffrey J. Warren, ${ }^{4}$ and James M. Mayer*,1 \\ ${ }^{1}$ Department of Chemistry, Yale University, New Haven, Connecticut 06520, United States \\ 2 Department of Chemistry, The College of New Jersey, Ewing, NJ 08628, United States \\ ${ }^{3}$ Department of Chemistry, University of British Columbia, Vancouver, BC, Canada V6T 1Z1 \\ ${ }^{4}$ Department of Chemistry, Simon Fraser University, Burnaby, BC, Canada V5A 1S6
}

Table of Contents



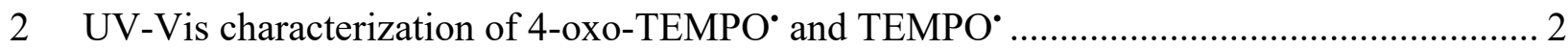

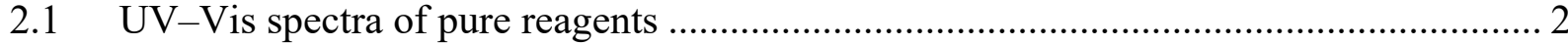

2.2 Molar absorptivities $(\varepsilon)$ of 4-oxo-TEMPO ${ }^{\bullet}$ and TEMPO` ............................................ 2



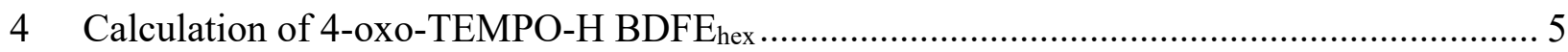

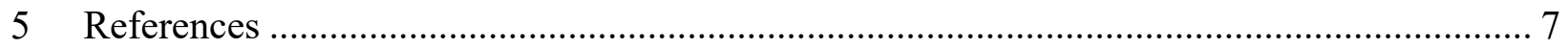




\section{General Considerations}

Reagents were purchased from Sigma-Aldrich or Alfa Aesar. TEMPO` and 4-oxo-TEMPO` were sublimated under vacuum prior to use. TEMPO-H was prepared as reported previously. ${ }^{1}$ Hexanes (mixture of isomers, HPLC grade) were purchased from Sigma-Aldrich and dispensed from an Argon-atmosphere solvent system with alumina drying columns. All reactions were performed in a nitrogen-filled glovebox (working $\mathrm{O}_{2}$ level $<5 \mathrm{ppm}$ ). UV-Vis spectra were collected on an Agilent Cary 60 UV-Vis or Agilent Cary 5000 UV-Vis-NIR spectrophotometer inside of a nitrogen-filled glovebox.

\section{UV-Vis characterization of 4-0xo-TEMPO` and TEMPO•}

\section{$2.1 \quad U V-V i s$ spectra of pure reagents}

Individual UV-Vis spectra were taken of 4-oxo-TEMPO` (oxo ${ }^{\circ}, \lambda_{\max }=459 \mathrm{~nm}$ ), TEMPO` $\left(\lambda_{\max }=473 \mathrm{~nm}\right)$, and TEMPO-H in hexane. The spectra were normalized to a concentration of 40 $\mathrm{mM}$ and are shown overlaid in Figure S1. TEMPO-H displayed a negligible UV-Vis trace, confirming it was free from TEMPO` contamination.

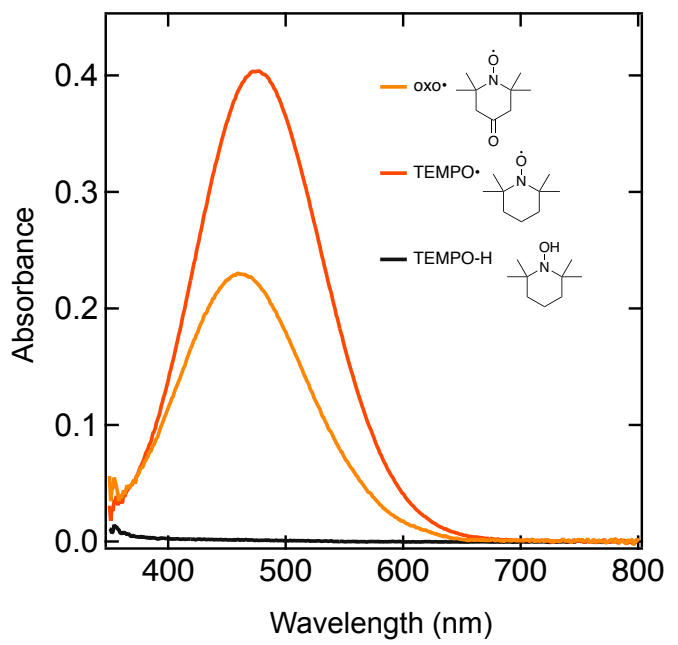

Figure S1. UV-Vis spectra of 40 mM oxo', TEMPO', and TEMPO-H in hexane.

\subsection{Molar absorptivities ( $\varepsilon$ ) of 4-oxo-TEMPO• and TEMPO•}

Beer's law plots were constructed for oxo (Figure S2a) and TEMPO`(Figure S2b) in hexane. The molar absorptivities were determined to be $\varepsilon 459 \mathrm{~nm}=5.86 \mathrm{M}^{-1} \mathrm{~cm}^{-1}$ and $\varepsilon 473 \mathrm{~nm}=10.27 \mathrm{M}^{-1} \mathrm{~cm}^{-1}$ for oxo $^{\circ}$ and TEMPO', respectively. These values were in good agreement with the experimental values for $\varepsilon \lambda_{\max }$ when $\varepsilon$ was calculated at each wavelength (Figure S2c). 



Figure S2. Concentration was plotted against absorbance at the $\lambda_{\max }$ for (A) oxo ${ }^{\circ}$ and (B) TEMPO $^{*}$ in hexane. The slope of the resulting line is equal to $\varepsilon_{\lambda \max }$ for each species. (C) The absorbance at each wavelength was divided by concentration of the sample to provide the epsilon at each wavelength. 


\section{UV-Vis Equilibration}

Scheme 1. Reaction between TEMPO-H and oxo $\cdot K_{\text {eq }}$ of this reaction is directly related to the relative bond strength of TEMPO-H and oxo-H.

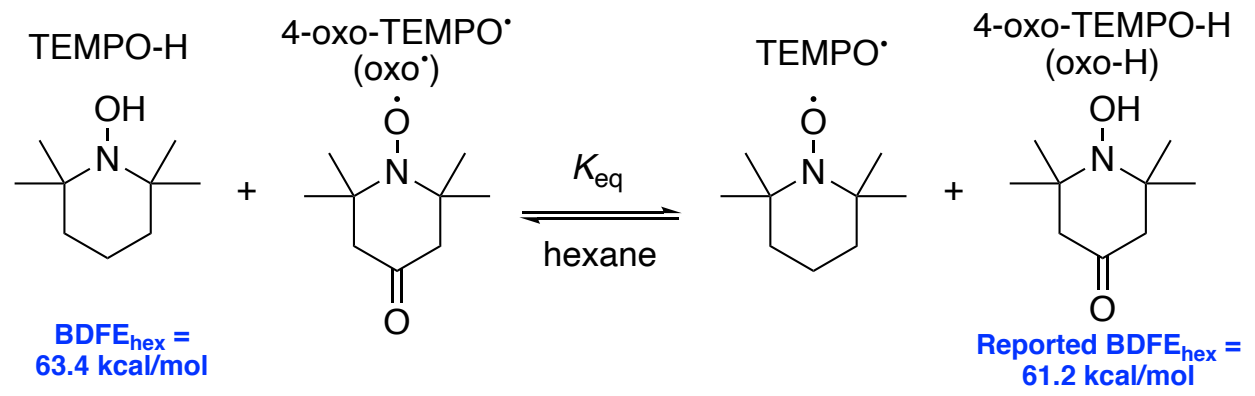

The pseudo-self exchange reaction between 4-oxo-TEMPO ${ }^{\circ}$ (oxo ${ }^{\circ}$ ) and TEMPO-H (Scheme 1) was analyzed to determine the bond dissociation free energy (BDFE) of 4-oxo-TEMPO-H (oxo$\mathrm{H})$ in hexanes. The equilibrium constant for this reaction, $K_{\text {eq }}$, gives the free energy of the reaction, $\Delta G^{\circ}$. This free energy relates the BDFEs of the H-atom donor, TEMPO-H, and the H-atom receptor, oxo ${ }^{\circ}$ eq $\quad \mathrm{X}-\mathrm{H}+\mathrm{Y} \rightarrow \mathrm{X}+\mathrm{Y}-\mathrm{H} \quad \Delta G^{\circ}=\mathrm{BDFE}(\mathrm{XH})-\mathrm{BDFE}(\mathrm{YH}) \quad$ (S1). Utilizing the known TEMPO-H BDFE hexane $^{2}=63.4 \mathrm{kcal} \mathrm{mol}^{-1}$, we were able to calculate the BDFE of oxo-H.

$$
\mathrm{X}-\mathrm{H}+\mathrm{Y} \rightarrow \mathrm{X}+\mathrm{Y}-\mathrm{H} \quad \Delta G^{\circ}=\operatorname{BDFE}(\mathrm{XH})-\mathrm{BDFE}(\mathrm{YH})
$$

A range of experimental initial concentrations were chosen for the equilibration reaction, ranging from 1:0.33 - 1:1.2 oxo ${ }^{\circ}$ TEMPO-H (Table $\mathrm{S} 1$ ). A $2 \mathrm{~mL}$ solution of $30 \mathrm{mM}$ oxo ${ }^{\bullet}$ in hexanes was prepared in a quartz cuvette equipped with a stir bar. TEMPO-H stock solution in hexane $(0.1933 \mathrm{M})$ was titrated incrementally across the range of oxo:TEMPO-H molar ratios. After each TEMPO-H addition, the reaction was equilibrated with stirring for 20 min until the UV-Vis trace was constant. Sequential addition of TEMPO-H stock solution and subsequent equilibration resulted in seven experimental equilibrations with UV-Vis spectra shown in Figure S3. Addition of TEMPO-H resulted in a slight red shift in $\lambda_{\max }$ and an increase in absorbance as more TEMPO was formed at equilibrium. 
Table S1. Experimental setup for seven equilibration reactions between oxo ${ }^{\bullet}$ and TEMPO-H. A solution of $\operatorname{oxo}^{\circ}(0.817 \mathrm{~mL}, 0.0734 \mathrm{M})$ was reacted with increasing amounts of TEMPO-H stock solution and equilibrated for $20 \mathrm{~min}$. [oxo $\left.{ }^{\circ}\right]_{\mathrm{i}}$ and $[\mathrm{TEMPO}-\mathrm{H}]_{\mathrm{i}}$ are initial concentrations of these species in solution.

\begin{tabular}{|c|c|c|c|c|c|c|c|}
\hline Sample & $\begin{array}{l}\text { oxo } \\
\text { equiv. }\end{array}$ & $\begin{array}{l}\text { TEMPOH } \\
\text { equiv. }\end{array}$ & $\begin{array}{c}\text { mmol } \\
\text { oxo }\end{array}$ & $\begin{array}{c}\text { mmol } \\
\text { TEMPOH }\end{array}$ & $\begin{array}{l}\text { Total volume } \\
\qquad(\mathrm{mL})\end{array}$ & {$\left[\mathrm{oxo}^{\circ}\right]_{\mathrm{i}}(\mathrm{mol} / \mathrm{L})$} & $\underset{(\mathrm{TEMPOH}]_{\mathrm{i}}}{[\mathrm{mol} / \mathrm{L})}$ \\
\hline $\mathrm{A}$ & 1.0 & 0.33 & 0.060 & 0.020 & 2.103 & 0.0285 & 0.0095 \\
\hline B & 1.0 & 0.50 & 0.060 & 0.030 & 2.289 & 0.0262 & 0.0131 \\
\hline $\mathrm{C}$ & 1.0 & 0.63 & 0.060 & 0.038 & 2.328 & 0.0258 & 0.0161 \\
\hline $\mathrm{D}$ & 1.0 & 0.71 & 0.060 & 0.043 & 2.356 & 0.0255 & 0.0182 \\
\hline E & 1.0 & 0.83 & 0.060 & 0.050 & 2.393 & 0.0251 & 0.0209 \\
\hline $\mathrm{F}$ & 1.0 & 1.0 & 0.060 & 0.060 & 2.444 & 0.0245 & 0.0245 \\
\hline G & 1.0 & 1.2 & 0.060 & 0.072 & 2.506 & 0.0239 & 0.0287 \\
\hline & & & .05 & 500 & 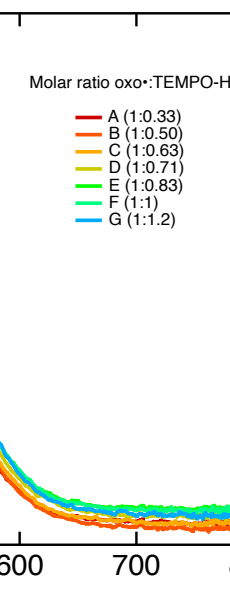 & & \\
\hline & \multicolumn{7}{|c|}{ Wavelength $(\mathrm{nm})$} \\
\hline
\end{tabular}

Figure S3. UV-Vis absorbances of oxo ${ }^{\bullet}+$ TEMPO-H reactions corrected against a hexane blank.

\section{Calculation of 4-oxo-TEMPO-H BDFE ${ }_{h e x}$}

The concentrations of each species - oxo', oxo-H, TEMPO', and TEMPO-H - can be calculated at equilibrium using the total absorbance, the molar absorptivities of the two colored species (oxo ${ }^{\circ}$ and TEMPO'), and the initial concentrations of oxo $^{\circ}$ and TEMPO-H. The total absorbance at each wavelength is the sum of the absorbance of each species (eq. S2). Mass balance dictates that the sum of the concentrations of related species $\left(\mathrm{oxo}^{\circ}+\mathrm{oxo}^{-\mathrm{H}}\right.$; $\mathrm{TEMPO}^{\circ}+\mathrm{TEMPO}^{-}$ $\mathrm{H}$ ) at equilibrium must be equal the initial concentration of the related reactant species (eqs. S3 and S4). Additionally, the concentration of the products oxo-H and TEMPO must be equivalent (eq. S5) in the absence of decomposition or side reactivity (of which none was observed by NMR or UV-Vis spectroscopies). 


$$
\begin{aligned}
& \mathrm{Abs}^{\lambda}=l\left(\varepsilon_{\mathrm{TEMPO}}^{\lambda}\left[\mathrm{TEMPO}^{\bullet}\right]+\varepsilon_{\mathrm{OxO}}^{\lambda} \cdot\left[\mathrm{OXO}^{\bullet}\right]\right) \\
& {\left[\mathrm{oxo}^{\bullet}\right]_{\mathrm{i}}=\left[\mathrm{oxo}^{\bullet}\right]+[\mathrm{oxo}-\mathrm{H}]} \\
& {\left[\text { TEMPO-H }_{\mathrm{i}}=[\text { TEMPO-H }]+\text { [TEMPO }^{\circ}\right]} \\
& {[\text { oxo-H }]=\left[\text { TEMPO }^{\circ}\right]}
\end{aligned}
$$

The system of equations above were solved for [oxo ${ }^{\circ}$ and [TEMPO ${ }^{\circ}$, resulting in equations S6 and S7.

$$
\begin{gathered}
{\left[\mathrm{oxo}^{\bullet}\right]=\frac{\left(\mathrm{Abs}^{\lambda} / l\right)-\varepsilon_{\left.\mathrm{TEMPO} \cdot\left[\mathrm{oxo}^{\bullet}\right]_{\mathrm{i}}\right)}^{\lambda}}{\varepsilon_{\mathrm{oxO} \bullet}^{\lambda} \cdot \varepsilon_{\mathrm{TEMPO} \cdot}^{\lambda}}} \\
{\left[\mathrm{TEMPO}^{\bullet}\right]=\frac{\left(\mathrm{Abs}^{\lambda} / l\right)-\varepsilon_{\mathrm{OxO} \bullet}^{\lambda}\left[\mathrm{oxo}^{\bullet}\right]}{\varepsilon_{\mathrm{TEMPO} \cdot}^{\lambda}}}
\end{gathered}
$$

For each sample A-G, the concentrations of all species at equilibrium were calculated from

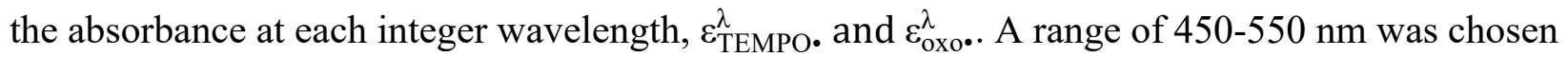
due to the relatively large difference in $\varepsilon$ between the species within these values. Representative data from sample B is provided in Table S2. At each wavelength (and for each sample) $K_{\text {eq }}$ was determined using the calculated concentrations of the species at equilibrium, yielding an average $K_{\text {eq }}=3.9 \pm 2.0$. This corresponds to $\Delta G^{\circ}=-0.8 \pm 0.4$. Utilizing the known BDFE hex $_{\text {of TEMPO-H }}$




Table S2. UV-Vis spectroscopy data and calculated concentrations of reaction species from wavelengths $550-450 \mathrm{~nm}$.

Sample B, calculated values

\begin{tabular}{|c|c|c|c|c|c|c|c|c|c|}
\hline $\begin{array}{l}\text { Wavelength } \\
(\mathbf{n m})\end{array}$ & $\varepsilon_{0 \times 0}$ & $\varepsilon_{\text {TEMPO }}$ & $\begin{array}{c}\text { Sample B, } \\
\text { raw Abs } \\
\text { data }\end{array}$ & $\begin{array}{c}\text { Sample B, } \\
\text { corrected } \\
\text { Abs data }\end{array}$ & {$\left[\mathbf{o x o}^{\circ}\right]$} & [TEMPO'] & [oxo-H] & [TEMPO-H] & $K_{\mathrm{eq}}$ \\
\hline 550 & 1.83 & 4.52 & 0.0765 & 0.0809 & 0.0167 & 0.0111 & 0.0111 & 0.00279 & 2.66 \\
\hline 545 & 2.06 & 5.02 & 0.0858 & 0.0901 & 0.0168 & 0.0110 & 0.0110 & 0.00288 & 2.52 \\
\hline 540 & 2.28 & 5.47 & 0.0945 & 0.0992 & 0.0167 & 0.0112 & 0.0112 & 0.00273 & 2.75 \\
\hline 535 & 2.58 & 6.01 & 0.105 & 0.109 & 0.0170 & 0.0109 & 0.0109 & 0.00304 & 2.29 \\
\hline 530 & 2.82 & 6.55 & 0.115 & 0.119 & 0.0169 & 0.0110 & 0.0110 & 0.00294 & 2.44 \\
\hline 525 & 3.03 & 7.05 & 0.126 & 0.130 & 0.0164 & 0.0114 & 0.0114 & 0.00249 & 3.19 \\
\hline 520 & 3.38 & 7.59 & 0.135 & 0.140 & 0.0170 & 0.0108 & 0.0108 & 0.00308 & 2.24 \\
\hline 515 & 3.69 & 8.09 & 0.147 & 0.151 & 0.0168 & 0.0111 & 0.0111 & 0.00287 & 2.53 \\
\hline 510 & 3.98 & 8.56 & 0.156 & 0.160 & 0.0170 & 0.0108 & 0.0108 & 0.00309 & 2.23 \\
\hline 505 & 4.30 & 8.97 & 0.166 & 0.170 & 0.0171 & 0.0108 & 0.0108 & 0.00316 & 2.15 \\
\hline 500 & 4.57 & 9.39 & 0.174 & 0.179 & 0.0170 & 0.0108 & 0.0108 & 0.00308 & 2.24 \\
\hline 495 & 4.83 & 9.71 & 0.183 & 0.187 & 0.0171 & 0.0107 & 0.0107 & 0.00321 & 2.08 \\
\hline 490 & 5.10 & 9.99 & 0.191 & 0.195 & 0.0170 & 0.0109 & 0.0109 & 0.00304 & 2.30 \\
\hline 485 & 5.32 & 10.2 & 0.197 & 0.202 & 0.0169 & 0.0109 & 0.0109 & 0.00298 & 2.38 \\
\hline 480 & 5.52 & 10.3 & 0.202 & 0.206 & 0.0168 & 0.0110 & 0.0110 & 0.00290 & 2.49 \\
\hline 475 & 5.66 & 10.3 & 0.206 & 0.210 & 0.0165 & 0.0113 & 0.0113 & 0.00261 & 2.97 \\
\hline 470 & 5.77 & 10.3 & 0.207 & 0.211 & 0.0167 & 0.0112 & 0.0112 & 0.00275 & 2.73 \\
\hline 465 & 5.83 & 10.1 & 0.206 & 0.211 & 0.0166 & 0.0113 & 0.0113 & 0.00266 & 2.87 \\
\hline 460 & 5.86 & 9.91 & 0.205 & 0.209 & 0.0164 & 0.0114 & 0.0114 & 0.00248 & 3.21 \\
\hline 455 & 5.76 & 9.57 & 0.203 & 0.206 & 0.0159 & 0.0120 & 0.0120 & 0.00194 & 4.65 \\
\hline 450 & 5.71 & 9.19 & 0.197 & 0.201 & 0.0157 & 0.0122 & 0.0122 & 0.00173 & 5.48 \\
\hline
\end{tabular}

\section{References}

1. Wu, A.; Masland, J.; Swartz, R. D.; Kaminsky, W.; Mayer, J. M., Synthesis and Characterization of Ruthenium Bis( $\beta$-diketonato) Pyridine-Imidazole Complexes for Hydrogen Atom Transfer. Inorg. Chem. 2007, 46 (26), 11190-11201.

2. Malievskii, A. D.; Koroteev, S. V.; Shapiro, A. B., Kinetics and thermodynamics of hydrogen atom exchange reactions in sterically hindered hydroxylamine-nitroxyl radical systems. Kinet. Catal. 2005, 46 (6), 812-820. 